\title{
Der Anfang - und wie es weiterging: 125 Jahre Klinisches Institut für Neurologie*
}

Im Jahr 2007 feierte das Klinische Institut für Neurologie der Medizinischen Universität Wien sein 125-jähriges Bestehen [1]. Anfänge sind immer von besonderem Interesse, jedoch wissen wir oft nicht, wie etwas angefangen hat - denken Sie etwa an die Entstehung unseres Universums. Das Klinische Institut für Neurologie ist natürlich nicht das Universum, aber, zumindest für uns, eine kleine, vertraute und geliebte Welt, von der wir wissen, wann und wie sie entstanden ist. Unser Urknall trägt den Namen Heinrich Obersteiner, und das erste Fassbare war ein Schrank - auf Wienerisch ein Kasten. Wie kam es dazu?

Heinrich Obersteiner (13. 11. 1847 - 19. 11. 1922; Abb. 1) stammte aus einer Arztfamilie [2]. Sein Vater war Leiter einer berühmten privaten Nervenheilanstalt im 19. Wiener Gemeindebezirk („Privat-Irren-Heilanstalt“, in der u. a. auch der Dichter Nikolaus Lenau untergebracht war, und die später vom Sohn bis ins hohe Alter weitergeführt wurde). Heinrich Obersteiner absolvierte 1865 das Schottengymnasium mit Auszeichnung, studierte Medizin an der Universität Wien, promovierte 1870 und arbeitete zunächst im neurohistologischen Laboratorium des Physiologen Ernst von Brücke, der Obersteiners Interesse für das Nervensystem in jeder Hinsicht förderte.

Im Jahr 1873 wurde Obersteiner Universitätsdozent für Anatomie und Pathologie des Nervensystems und 1880 Extraordinarius für Physiologie und Pathologie des Nervensystems.

Im Jahr 1882 reichte Obersteiner bei der Behörde ein Gesuch um Überlassung eines Raumes zwecks Gründung eines eigenen Instituts für sein Arbeitsgebiet ein. In der Beantwortung dieses Gesuches hieß es lapidar: „Damit Euer Wohlgeboren jedoch die nötigen Präparate und Apparate dauernd aufbewahren können, wird die Aufstellung eines geräumigen Kastens hinreichen, zu welchem Ende Sie sich mit der einen oder anderen der mit den Lokalitäten im genannten Institute (Pathologie) vertrauten Personen ins Einvernehmen setzen wollen ..."

Die Existenz des Instituts und seine Tätigkeit wurde materiell nur durch Obersteiners selbstloses Mäzena-

\footnotetext{
* Nach einem Vortrag am 23. November 2007 zur Feier des 125-jährigen Bestehens des Klinischen Instituts für Neurologie der Medizinischen Universität Wien.

Korrespondenz: Em. Univ.-Prof. Dr. H. Bernheimer, Klinisches Institut für Neurologie der Medizinischen Universität Wien, Allgemeines Krankenhaus 4J, Postfach 48, 1097 Wien, Österreich, E-mail: hans.bernheimer@meduniwien.ac.at
}

tentum ermöglicht: er stellte dafür in nobler, großzügiger Weise seine eigenen finanziellen Mittel bereit (wir würden heute vielleicht sagen als „Auto-Fundraising“; die Obersteiner zugestandene staatliche Dotation hätte kaum für die Kosten des Präparatespiritus ausgereicht). Das Institut (zur Zeit seiner Gründung „Universitätsinstitut für Anatomie und Physiologie des Centralnervensystems“, ab 1900 „Neurologisches Institut“, ab 1993 „Klinisches Institut für Neurologie“ genannt) war für lange Zeit in verschiedenen, fast ruinenhaften Räumen untergebracht (Abb. 2); nach diversen Umsiedlungen fand sich erst im Jahr 1917 eine eigene Unterkunft in der Schwarzspanierstraße 17. Das Institut war nicht nur die erste, sondern für viele Jahre auch die einzige Lehr- und Forschungsstätte der „Neurosciences“ in Europa und den USA und wurde Ausgangspunkt der Errichtung von Hirnforschungsinstituten in der ganzen Welt, wie z.B. in Philadelphia, Madrid, Zürich, Amsterdam, Frankfurt etc.

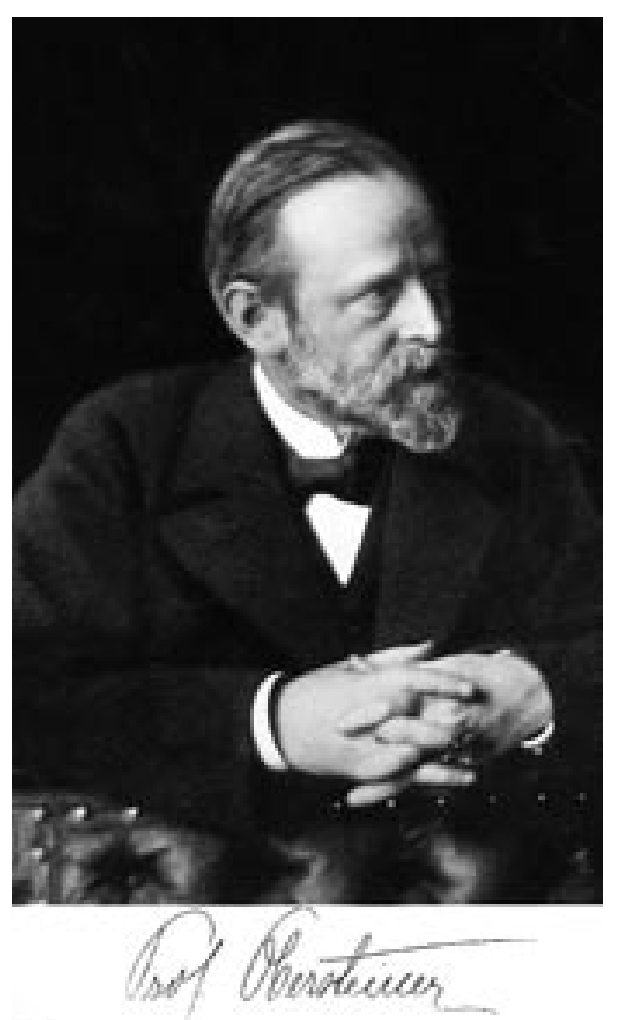

Abb. 1. Heinrich Obersteiner in mittleren Jahren 


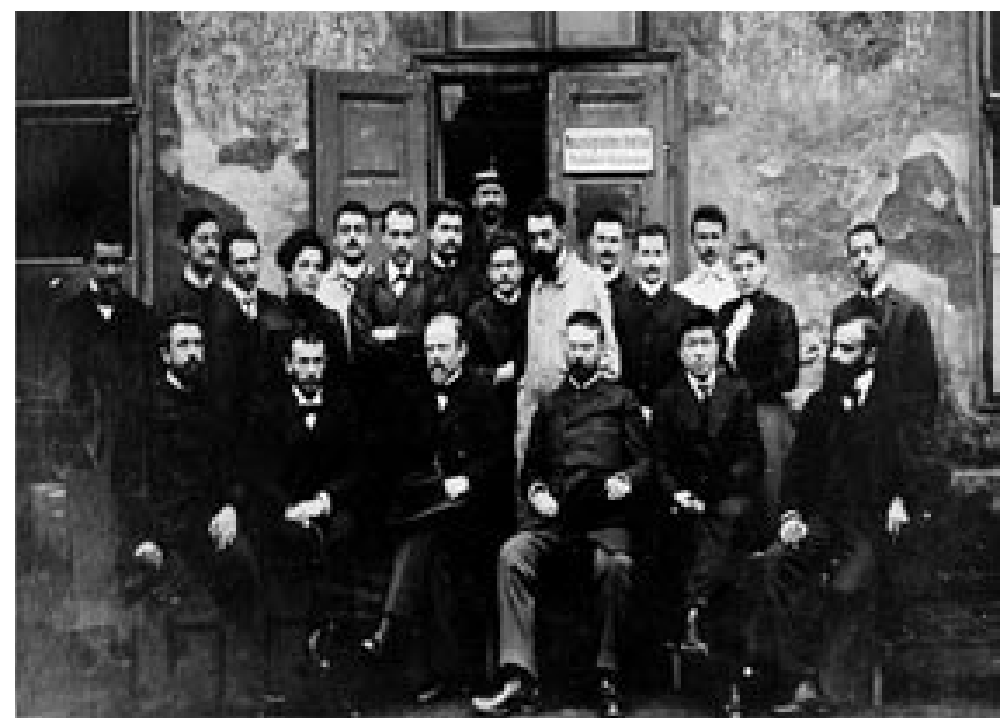

Abb. 2. Heinrich Obersteiner, Mitarbeiter und Schüler vor dem Eingang in das Neurologische Institut in der „alten Gewehrfabrik“. Heinrich Obersteiner sitzend in der 1. Reihe, Dritter von links; rechts hinter ihm stehend im hellen Arbeitsmantel sein späterer Nachfolger Otto Marburg

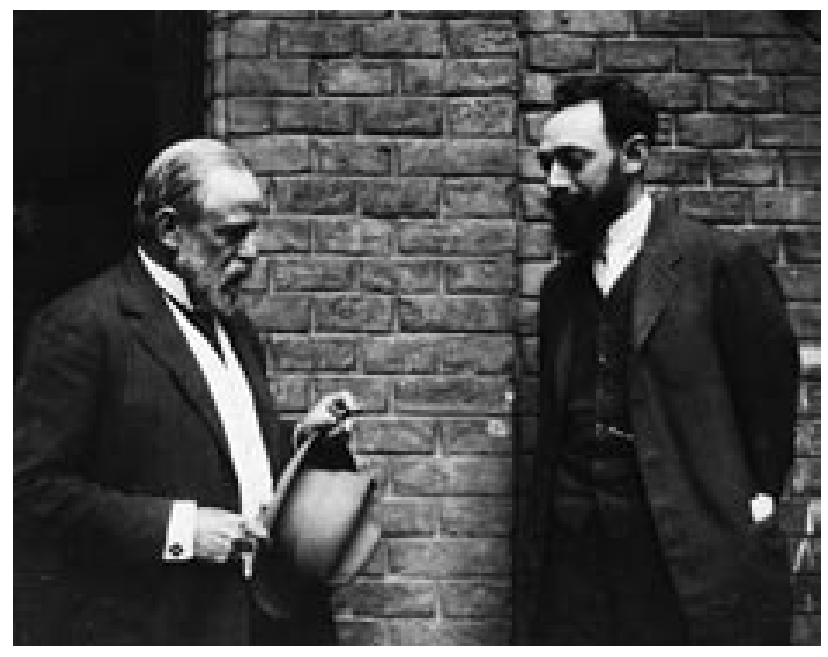

Abb. 3. Heinrich Obersteiner (links) und Otto Marburg

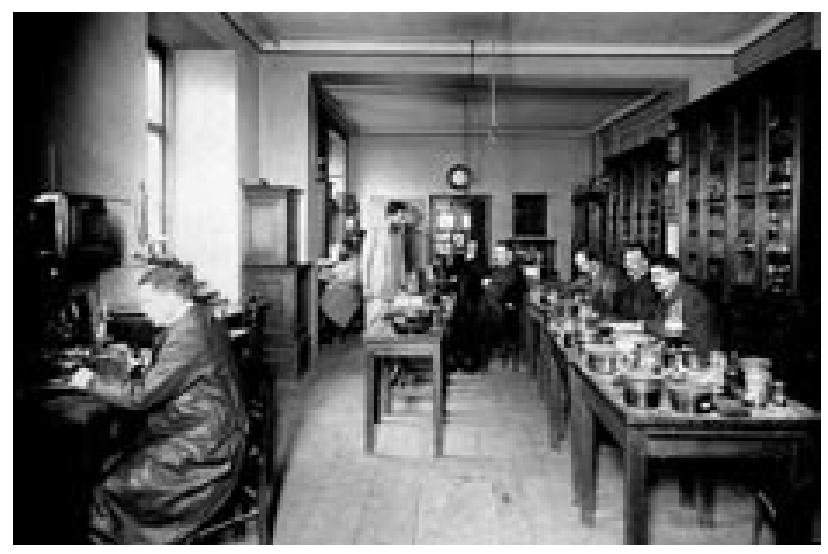

Abb. 4. Arbeitsraum im Neurologischen Institut. Otto Marburg stehend im hellen Arbeitsmantel und Studierende
Obersteiner fasste das gesamte Wissensgut seiner Zeit zusammen. Sein Lehrbuch „Anleitung beim Studium des Baues der nervösen Zentralorgane" wurde von Otto Marburg, dem Mitarbeiter und Nachfolger Obersteiners, als „Lehrbuch der ganzen Welt“ bezeichnet. Darüber hinaus war Obersteiner Herausgeber der legendären Zeitschrift „Arbeiten aus dem Neurologischen Institut der Universität Wien“ und übergab der Bibliothek des Neurologischen Instituts seine eigene umfangreiche wissenschaftliche Spezialbibliothek (mehr als 40.000 Bände) als Schenkung.

Obersteiner war Autor essentieller Beiträge zur Neuroanatomie, Neuropathologie und Neurologie und war im Grunde genommen der Urvater der „Neurosciences“, der Neurowissenschaften. In diesem Forschungsbereich kommt es nicht so sehr auf die Mehrzahl von Disziplinen im Sinne einer Pluri- oder Multidisziplinität, sondern auf die konstruktive Zusammenarbeit unterschiedlicher Disziplinen im Sinne einer Syn-Disziplinarität [3] zur Entwicklung einer maximalen Effizienz an.

Die Lehrtätigkeit Obersteiners war Anziehungspunkt für zahlreiche Schüler aus dem In- und Ausland, nicht zuletzt aus Japan. Obersteiner schrieb über den Lehrbetrieb: „Jeder, der als Mitarbeiter ins Institut eintritt, ob Student in den ersten Jahren, oder als erfahrener Arzt, soll, wenn er nur das ehrliche Bestreben zeigt $\mathrm{zu}$ lernen und $\mathrm{zu}$ arbeiten, sich in einem Kreise von Freunden befinden. Darum habe ich auch von Anfang an mein Augenmerk darauf gerichtet, dass dieses Verhältnis zwischen Lehrern und Schülern und zwischen diesen beiden untereinander, ein ungetrübtes sei und bleibe; lieber einmal ein paar heitere fröhliche Worte mitten in die ernste Arbeit eingestreut, als Streit, Zank und wissenschaftlicher Neid“ [4].

Obersteiner emeritierte 1919 und verstarb 1922. Sein Nachfolger war sein langjähriger Mitarbeiter Otto Marburg (25. 5. 1874 - 13. 6. 1948; Abb. 3 und 4), dem in 
diesem Heft der Wiener Klinischen Wochenschrift eine eigene Arbeit gewidmet ist [5]. Marburg forcierte die Kontakte zur Klinik, vor allem zur Neurochirurgie, und übte eine wichtige Konsiliartätigkeit aus (das Neurologische Institut hatte lange Zeit keinen Zugang zum Autopsiematerial klinisch-neurologischer Patienten und war auf Einsendungen von außeruniversitären Spitälern angewiesen!).

Marburgs Tätigkeit am Neurologischen Institut fand durch die Annexion Österreichs im März 1938 durch den Nationalsozialismus ein jähes Ende. $\mathrm{Zu}$ diesem Zeitpunkt wurden an der Universität „die am 1. März 1938 im Dienst befindlich gewesenen Hochschullehrer und Assistenzkräfte entfernt oder ausgeschieden, weil sie jüdischer Abstammung oder jüdische Mischlinge oder jüdisch versippt oder mit jüdischen Mischlingen versippt waren“ - Ereignisse, die uns alle heute noch betroffen machen. Marburg emigrierte in die Vereinigten Staaten und verstarb 1948.

Die weitere Entwicklung des Neurologischen Instituts soll und kann hier nur kurz gestreift werden. Nach den unruhigen Kriegs- und Nachkriegszeiten übernahm der 1938 aus Österreich vertriebene und 1949 nach Wien zurückgekehrte Neurologe und Psychiater Hans Hoff (11.12.1897-23.8. 1969) die Leitung des Neurologischen Instituts [6]. Hoff war zur gleichen Zeit auch Vorstand der Wiener Universitätsklinik für Neurologie und Psychiatrie und verkörperte eine enge Verbindung zwischen den beiden Disziplinen. Er „vermenschlichte“ die Psychiatrie und erlangte bei der Wiener Bevölkerung eine spezielle Popularität: die Wiener sagten zu einem von ihnen für geistesgestört Befundenen nicht „Du bist verrückt“, sondern „Du gehörst zum Hoff“ ...

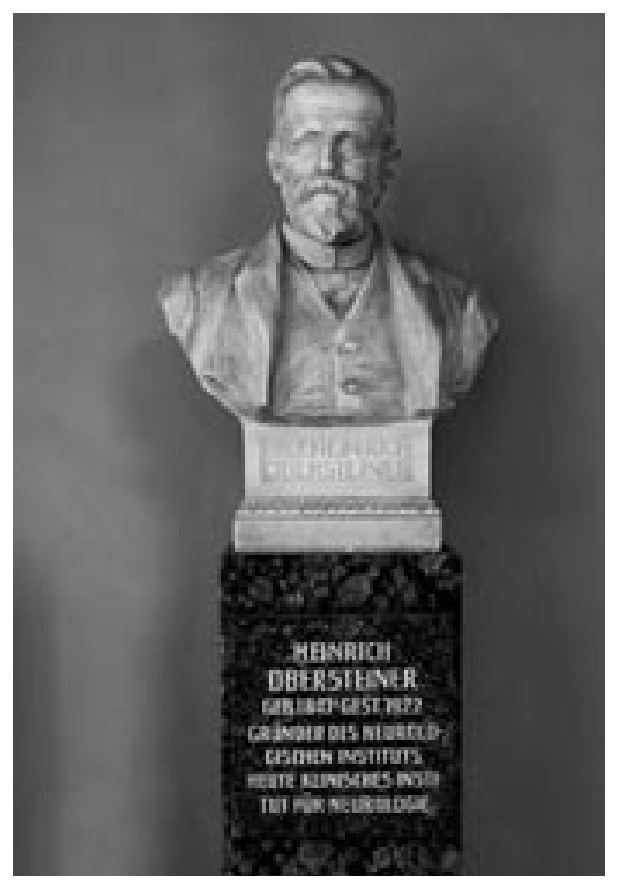

Abb. 5. Büste von Heinrich Obersteiner im Arkadenhof der Universität Wien
Hoffs Nachfolger Franz Seitelberger (4.12.1916-2. 11. 2007) kam in einer Zeit (1951) an das Neurologische Institut, als das Institut durch die Kriegs- und Nachkriegswirren völlig im Argen lag. Es ist ausschließlich Seitelbergers unermüdlichem Einsatz und seiner konsequenten Arbeit zu verdanken, dass das Institut wiederum zu einem national und international renommierten wissenschaftlichen Zentrum aufgebaut werden konnte. Die Mitarbeiter des Neurologischen Instituts in dieser Periode können hier nur kurz und inkomplett angeführt werden, wie Kurt Jellinger (Neuropathologie), Herbert Budka (Neuropathologie, seit 1998 Vorstand des Klinischen Instituts für Neurologie), Hellmut Petsche (Neurophysiologie, Enzephalographie), Elfriede Sluga (Nerven- und Muskelpathologie, Elektronenmikroskopie), Gustav Lassmann (Pathologie des autonomen Nervensystems), Karl Gloning (Neuropsychologische Arbeitsrichtungen, Störungen der höheren Hirnleistungen), Hans Lassmann (Experimentelle Neuropathologie, Multiple Sklerose; Direktor des Instituts für Hirnforschung der Medizinischen Universität Wien 19992007), und Hans (Hanno) Bernheimer, Verfasser der vorliegenden Publikation (Neurochemie; Vorstand des Klinischen Instituts für Neurologie 1987-1998).

Seitelbergers Intention war letztlich, eine differenzierte Integration von theoretischen und klinischen Neurowissenschaften (Neurologie, Psychiatrie, Neuropathologie, Neurochemie, Neurophysiologie etc.) ins Leben zu rufen - ein damals in Wien sehr schwieriges, aber heute selbstverständliches Strukturkonzept. Franz Seitelberger bekleidete auch die Ämter des Dekans der Medizinischen Fakultät sowie des Rektors der Universität Wien [7].

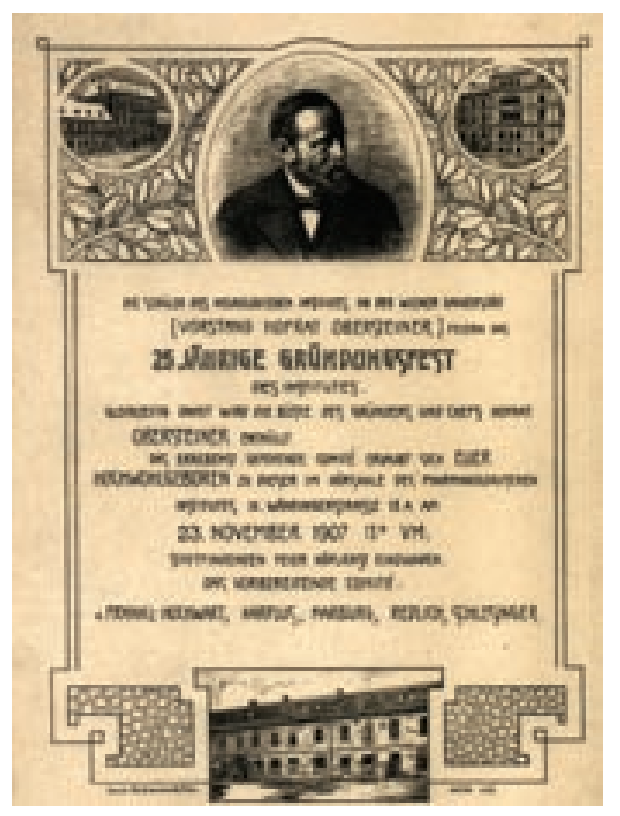

Abb. 6. Einladung zum 25-jährigen Gründungsfest des Neurologischen Instituts am 23. November 1907 
Abschließend nochmals zu Obersteiner. Wir freuen uns sehr, dass wir vor rund 10 Jahren mit Hilfe von Magnifizenz Schütz - damals Dekan der medizinischen Fakultät der Universität Wien, seit 2004 Rektor der nun selbstständigen Medizinischen Universität Wien - die Marmorbüste Obersteiner (Abb. 5), die sich im Neurologischen Institut in der Schwarzspanierstraße befand, in den Arkadenhof der Universität Wien übersiedeln konnten. Diese Büste war anlässlich des 25-jährigen Gründungsfests des Neurologischen Instituts am 23. November 1907 enthüllt worden (Abb. 6).

Obersteiner hatte einen Wahlspruch für das Neurologische Institut: „Ignorabimus, tamen impavidi progrediamur" (Wir werden es nicht wissen, aber dennoch unerschrocken fortschreiten). Ich möchte diesem Wahlspruch einen Gedanken des portugiesischen Dichters Fernando Pessoa hinzufügen: „Wir wissen nichts - und Phantasie ist alles!“ Vielleicht, im Sinne Obersteiners, ein Schritt zu neuen Erkenntnissen ...

Hans (Hanno) Bernheimer

\section{Danksagung}

Ich danke Frau Magistra Michaela Zykan und Frau Brigitte Maurer, Bildarchiv, Sammlungen der Medizinischen Universität Wien, für die freundliche Bereitstellung der Abbildungen 1-4 und 6, und Andreas Jurkowitsch am Klinischen Institut für Neurologie der Medizinischen Universität Wien für das Bild des Heinrich
Obersteiner-Denkmals (Abb. 5). Ganz besonders danke ich Univ.-Prof. Dr. Dr. h.c. Herbert Budka und A.o. Prof. Dr. Johannes Hainfellner, Klinisches Institut für Neurologie der Medizinischen Universität Wien, für die zahlreichen anregenden Gespräche.

\section{Literatur}

1. Kreft G, Kovacs G, Voigtländer T, Haberler C, Hainfellner JA, Bernheimer H, Budka H (2008) 125th Anniversary of the Institute of Neurology (Obersteiner-Institute) in Vienna. „Germ Cell“ of Interdisciplinary Neuroscience. Clinical Neuropathology (im Druck)

2. Hoff H, Seitelberger F (1962) Heinrich Obersteiner. Zur 40. Wiederkehr seines Todestages im 80. Gründungsjahr des Neurologischen Instituts. Wien Klin Wochenschr 74: 682684

3. Hainfellner J, Bernheimer H (2007) Bessere Bezeichnungen. Die Presse, S 30

4. Obersteiner H (1919) Rückschau - Ausblick. Arbeiten aus dem Neurologischen Institute (österreichisches interakademisches Zentralinstitut für Hirnforschung) an der Wiener Universität XXII: 557-572

5. Triarhou LC (2008) Professor Otto Marburg (1874-1948), universal neurologist and the „dean of teachers“. Wien Klin Wochenschr (im Druck)

6. Bernheimer H (1993) Editorial. Neuropsychiatrie 7: 1-5

7. Seitelberger F (1995) Das Neurologische Institut (Obersteiner-Institut) der Universität Wien. Geschichte, Entwicklung, gegenwärtige Situation. Neuropsychiatrie 9: $75-81$

Weitere Literatur beim Autor 